\title{
Distributed Coding Modulation Adaptation Scheme for Relay Channel
}

\author{
Zi Teng ${ }^{1,2}$, Jun $\mathrm{Wu}^{{ }^{*}}$, Min Wang ${ }^{1,3}$, Lifeng Su${ }^{1}$ \\ ${ }^{1}$ College of Electronics and Information Engineering, Tongji University, Shanghai, China, \\ ${ }^{2}$ School of Mathematics, Physics \& Information Engineering, Jiaxing University, Jiaxing, China, \\ ${ }^{3}$ School of Mathematics and Computer Science, Gannan Normal University, Ganzhou, China. \\ Email: 2011tengz@tongji.edu.cn,wujun@tongji.edu.cn,2011mwangcs@tongji.edu.cn, sulifeng@tongji.edu.cn
}

Received May, 2013

\begin{abstract}
Rate adaptation is an effective approach to achieve high spectrum efficiency under varying channel condition, especially for wireless communication. This paper proposes rate adaptation at receiver for wireless relay system. In this scheme, source node uses a new modulation technology, called random projections code (RPC), to achieve rate adaptation. Both relay node and destination node decode the received RPC encoding signals. If destination does not decode RPC correctly, relay node will act compressing and forwarding role by performing LDPC syndrome encoding and sending syndrome coded information to destination node. We discuss how to jointly decode at destination node when it receives RPC coded information from source node and syndrome coded information from relay node. Finally, we evaluate the scheme by bit-error-rate (BER) and good put evaluation metrics. Simulation results show that the coding gain is about $4 \mathrm{~dB}, 3.1 \mathrm{~dB}, 2.2 \mathrm{~dB}$ and $1.6 \mathrm{~dB}$ for LDPC coding rate $0.8,0.89,0.94,0.99$ at BER $10^{-5}$ respectively. The throughput of the schemes is at least $0.3 \mathrm{bit} / \mathrm{s} / \mathrm{Hz}$ higher than RPC at SNR ranging from $5 \mathrm{~dB}$ to $25 \mathrm{~dB}$.
\end{abstract}

Keywords: Rate Adaptation; Random Projection Code; LDPC; Relay Channel

\section{Introduction}

Rate adaptation is critical to the performance of modern wireless communication system, e.g., WiFi, $3 \mathrm{G}$ and LTE. Conventional rate adaptation technology is implemented at sending end, and achieved by adjusting actual transmission rate through channel coding rate and modulation scheme[1,2], based on the current channel state information (CSI) [3]. However the existing approaches for rate adaptation at sending end have two defects. One is that it is always difficult to estimate CSI accurately due to feedback delay in frequency division duplex (FDD) system, because the channel may vary drastically during several data packets transmission. The other is that the transmission rate can be adjusted among limited modulation coding schemes (MCS), so the coarse granularity of MCS results in a staircase of spectrum efficiency.

To solve these problems mentioned above, three new schemes [4-6] have been proposed for smooth rate adaptation, and all of them implement rate adaptation at receiver and attain continuous spectrum efficiency. Cui et al. [4] has proposed the random projection code (RPC), which is a novel rate compatible modulation (RCM) technology, and modulated symbols are incrementally generated from information bits through weighted mapping. Compared to LDPC $[7,8]$, probability convolution operation is used in RPC for horizontal iteration instead of $\log (\tan h)$ operation. This is due to the fact that RPC symbols are generated using the arithmetic weighted sum instead of logical exclusive-OR (XOR). RPC combines channel coding and modulation together. In addition, Reference [4] has mentioned that a high coding-rate LDPC code serially concatenated with RPC can gain better performance, but no detailed performances are given.

On the other hand, how to use RPC in relay channel is a relative new topic. For traditional relay system, some researchers have proposed distributed channel coding (DCC) schemes for the three node relay channel to jointly optimize coding design at source node and relay node. These DCC schemes include distributed turbo codes (DTC) and distributed LDPC codes (DLDPC) [9]. References [10-14] have discussed how to design LDPC code for DF relay scheme. If the signal from relay node to destination node is compressed, those DLDPC models can be thought as typical distributed source coding (DSC). There are two major kinds of approach, i.e., parity approach and syndrome approach, to realize DSC $[15,16]$. Some researchers have proved that under ideal channel condition syndrome approach has the optimality, but under the noisy channel condition parity approach's performance is the best $[16,17]$. 
Inspired by DCC, DSC and potential coding gain brought by serially concatenating LDPC with RPC, we propose concatenating LDPC with RPC in parallel, and apply this parallel concatenation scheme to relay system. This scheme also has implemented rate adaptation at receiver with the help of LDPC coding at relay node. To our knowledge, the parallel concatenation of LDPC and RPC, and applying it to rate adaptation in relay system are seldom seen in publication.

The relay system consists of three nodes, i.e., one source, one relay and one destination. In our scheme, source node doesn't need CSI, except the ACK signal from destination node. Relay node and destination node achieve rate adaptation by using RPC. Relay node uses syndrome encoder to generate syndrome bits which are modulated by BPSK, and then transmits the modulation symbols to destination node. In destination node, we adopt joint decoder which needs two input data, i.e., the received RPC encoded information from source node and received syndrome information from relay node. Compared to pure RPC scheme, simulation results show that the coding gain is about $4 \mathrm{~dB}, 3.1 \mathrm{~dB}, 2.2 \mathrm{~dB}$ and $1.6 \mathrm{~dB}$ for coding rate $0.8,0.89,0.94$ and 0.99 at BER 10-5, the proposed schemes can enhance channel capacity at least $0.3 \mathrm{bit} / \mathrm{s} / \mathrm{Hz}$ at signal-to-noise ratio (SNR) ranging from 5 $\mathrm{dB}$ to $25 \mathrm{~dB}$. The simulation also indicates that the gain is insensitive to distance of relay node to source node and destination node.

The structure of this paper is organized as follows. Section 2 gives the system model of our relay scheme. Section 3 presents the processing procedure of source node and relay node. Section 4 presents how to decode jointly at destination node. The simulation results of our relay schemes are shown in section 5. Finally, Section 6 concludes this paper.

\section{System Model}

Our system model is depicted as Figure 1. We can see that this system includes three nodes, i.e., source node denoted as S, relay node denoted as R, and destination node denoted as D. There exists three channel links, i.e., SD link, SR link and RD link. The distance between the source and the destination is denoted by $d_{S D}, d_{S R}$ standing for the distance between the source and the relay, and $d_{R D}$ standing for the distance between the relay and the destination. Those distances satisfy the condition, i.e., $d_{S D}>d_{S R}$ and $d_{S D}>d_{R D}$.

We assume that relay node operates in a half-duplex mode. AWGN channel model is used for all the links, denoted as $N_{S R}, N_{R D}$ and $N_{R D}$ for the SR, RD and SD links, and their noise powers are $\sigma_{S R}^{2}, \sigma_{R D}^{2}$ and $\sigma_{S D}^{2}$, respectively. Setting radio signal propagation attenuation factor as $\alpha$, the relationships between the noise powers of different links can be given by

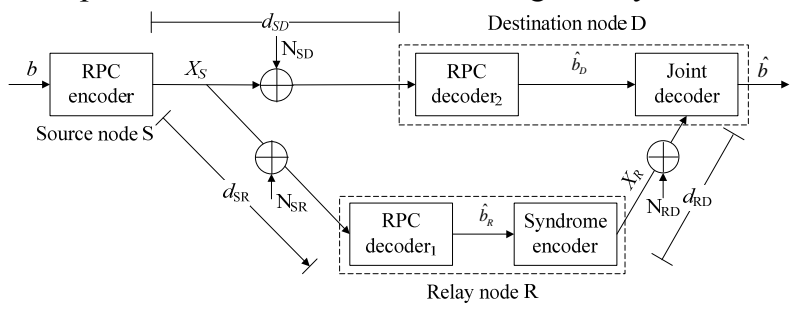

Figure 1. System model of the relay scheme.

$$
\frac{\sigma_{S R}^{2}}{\sigma_{S D}^{2}}=\left(\frac{d_{S R}}{d_{S D}}\right)^{\alpha} \text { and } \frac{\sigma_{R D}^{2}}{\sigma_{S D}^{2}}=\left(\frac{d_{R D}}{d_{S D}}\right)^{\alpha}
$$

Let $\alpha=2$ in this paper. Alternatively, the SNR of the different links are given by

$$
\begin{gathered}
S N R_{S R}=\frac{1}{\sigma_{S D}^{2}} \\
S N R_{S R}=\frac{1}{\sigma_{S R}^{2}}=S N R_{S D}\left(\frac{d_{S D}}{d_{S R}}\right)^{\alpha} . \\
S N R_{R D}=\frac{1}{\sigma_{R D}^{2}}=S N R_{S D} \cdot\left(\frac{d_{S D}}{d_{R D}}\right)^{\alpha} .
\end{gathered}
$$

$S$ uses RPC to encode $b$, and each two consecutive symbols are composed one modulation signal denoted as $\boldsymbol{X}_{S}$ Then $S$ broadcasts $\quad \boldsymbol{X}_{S}$ to $R$ and $D$. The relay node $R$ uses RPC decoder 2 to demodulate the signal from source node at first, and gets the information bits denoted as $\hat{\boldsymbol{b}}_{R}$. Then, $R$ re-encodes $\hat{\boldsymbol{b}}_{R}$ by LDPC encoder, and modulates parity encoded bits by BPSK to generate signal denoted as $\boldsymbol{X}_{\boldsymbol{R}} \cdot R$ transmits $\boldsymbol{X}_{R}$ to $D$. Finally, $D$ performs a concatenated decoding. After receiving the signal $X_{R}+N_{S D}$ from $S, D$ uses RPC decoder1 to demodulate signal, and gets the soft information $\hat{\boldsymbol{b}}_{\boldsymbol{D}}$. In the next time slot, $D$ receives parity encoding information from relay node, and then $D$ uses $\hat{\boldsymbol{b}}_{\boldsymbol{D}}$ as side information, performing LDPC decoding. In order to improve performance, both side information and parity information are used in soft information mode.

\section{Transmitter}

This system can be modeled as the time division relay channel. The total time is divided into three time slots, i.e., $t_{1}, t_{2}$, and $t_{3}$, for source node, relay node, and destination node respectively. In this section, we describe the detailed process of the source node and relay node.

\subsection{Source Encoding and Broadcasting}

The encoding principle of RPC is represented by a bipartite graph shown in Figure 2. Cycles denote bits nodes, and squares denote symbols nodes. A bipartite graph 
denoted as $G=(U, V, E)$. Here $U=\left\{b_{j}\right\}, j=1, \ldots, N$ is

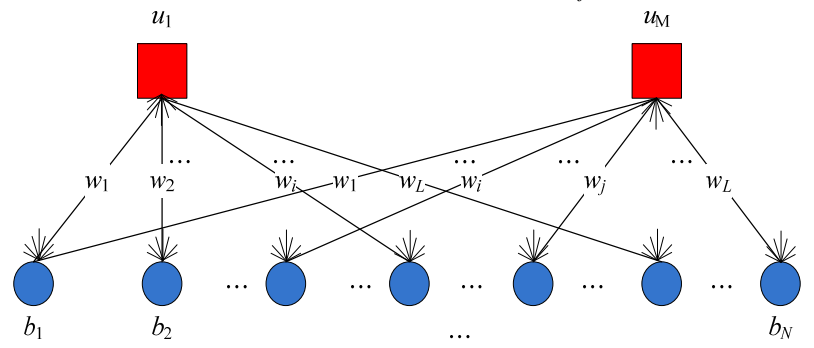

Figure 2. The bit-to-symbol mapping graph of RPC.

the set of bits nodes, $V=\left\{u_{i}\right\}, i=1, \ldots, M$ is the set of symbols nodes, and $E$ defines the connections between bit nodes and symbols nodes. A weight corresponding to an edge belongs to the weight set $w=\left\{w_{1}, w_{2}, \cdots, w_{L}\right\}$, and expresses energy proportion to symbol node for a corresponds bit node. The connections between bits and symbols can also be described by a $M \times N$ mapping matrix $\boldsymbol{G}$.

Given a bit block $\boldsymbol{b}=\left(b_{1}, b_{2}, \cdots, b_{N}\right)$, only the bits with these indices $i_{l}(l=1,2, \ldots, L)$ are sampled by a modulated symbol, so RPC modulated symbol can be written as

$$
u_{i}=\sum_{l=1}^{L} w_{l} \cdot b_{i_{l}}
$$

where $L$ is the number of non-zeros entities in $i-$ th row of $\boldsymbol{G}$, and $b_{i_{1}}$ is signal bits. RPC encodes b into a series of symbols by

$$
\boldsymbol{U}=\boldsymbol{G} \cdot \boldsymbol{b},
$$

where $\boldsymbol{U}$ is the generated symbols block for transmission.

RPC encoding and broadcasting takes place in time slot $t_{1}$. After $S$ is encoded into $\boldsymbol{U}$, each two consecutive symbols $\boldsymbol{U}$ are composed as one modulation signal given by

$$
U(2 k)+\sqrt{-1} \cdot U(2 k+1),\left(k=0,1, \cdots, \frac{N}{2}-1\right) .
$$

Finally, $S$ broadcasts $X_{S}$ to $R$ and $D$.

\subsection{Relay Node Decoding and Compress Forward}

Relay node performs RPC decoding, LDPC encoding and compressing forward in time slot $t_{2}$. $R$ first receives signal $\boldsymbol{Y}_{S R}$ from $\mathrm{S}$, and $\boldsymbol{Y}_{S R}$ is given by

$$
Y_{S R}=X_{S}+N_{S R}
$$

where $N_{S R}$ is noise term of channel SR, and $N_{S R} \sim$ $N\left(0, \sigma_{S R}^{2}\right)$. Then, R performs RPC decoding, and gets

$$
\hat{\boldsymbol{b}}_{\boldsymbol{R}}=\underset{\mathrm{b} \in \mathrm{GF}\left(2^{\mathrm{N}}\right)}{\operatorname{argmax}}\left\{\mathrm{P}\left(\boldsymbol{b} \mid \boldsymbol{Y}_{\boldsymbol{S R}}\right)\right\},
$$

where $\hat{\boldsymbol{b}}_{\boldsymbol{R}}$ is the results of hard decision of original RPC decoding algorithm.

After decoding, R uses LDPC parity check matrix $\boldsymbol{H}$ to encode $\hat{b}_{\boldsymbol{R}}$ again,

$$
\boldsymbol{P}_{R}=\mathrm{H}^{*} \hat{\boldsymbol{b}}_{\boldsymbol{R}},
$$

where $\boldsymbol{P}_{\boldsymbol{R}}$ is the parity bits. Then, $\mathrm{R}$ modulates $\boldsymbol{P}_{\boldsymbol{R}}$ with BPSK to form modulation signal $\boldsymbol{X}_{R}$, which is transmitted to D. Because of only transmitting the parity bits, $R$ acts as compressing and forwarding role.

\section{Jointly Decoding at Destination}

In this section, we mainly focus on the joint decoding at $D$ by using RPC and LDPC. And, we will analysis the feature of this joint decoding.

\subsection{Joint Decoding}

The diagram of joint decoding is depicted as Figure 3. From $y$-axis, joint decoding at $D$ is divided into two stages, one is RPC decoding, and the other is LDPC decoding. We use the original RPC decoding algorithm in joint decoding algorithm, but modify the hard decision part to output soft information as one part of input of LDPC decoding. The soft information correspond the information bits nodes from $b_{1}$ to $\boldsymbol{b}_{N_{1}}$, and can be regarded as the side information in DSC. In addition to information bits, there exist parity bits in $x$-axis, i.e., bits node from $\boldsymbol{b}_{N_{1}+1}$ to $\boldsymbol{b}_{N 2}$, which corresponds the channel prior information from relay node, just like compressing information in DSC.

$\mathrm{D}$ performs decoding in two consecutive time slots, i.e., $t_{2}$ and $t_{3}$. In time slot $t_{2}, D$ receives the signal denoted as $\boldsymbol{Y}_{S D}$ from source node $\mathrm{S}$, and $\boldsymbol{Y}_{S D}$ can be expressed as

$$
\boldsymbol{Y}_{S D}=\boldsymbol{X}_{S}+N_{S D}
$$

Then, $D$ performs RPC decoding algorithms to get soft informations denoted as $\hat{\boldsymbol{b}}_{\boldsymbol{D}}$,

$$
\hat{\boldsymbol{b}}_{\boldsymbol{D}}=\left(\hat{\boldsymbol{b}}_{\boldsymbol{D}}^{\mathbf{0}}, \hat{\boldsymbol{b}}_{\boldsymbol{D}}^{\mathbf{1}}\right)=\underset{b \in G F\left(2^{\mathrm{N}}\right)}{\operatorname{argmax}}\left\{\mathrm{P}\left(\boldsymbol{b} \mid \boldsymbol{Y}_{S D}\right)\right\}
$$

Here, we modify the hard decision part of RPC decoding algorithm proposed in [4]. Modified RPC decoding algorithm does not estimate the hard decision of input binary digit, but outputs the probability of corresponding bits.

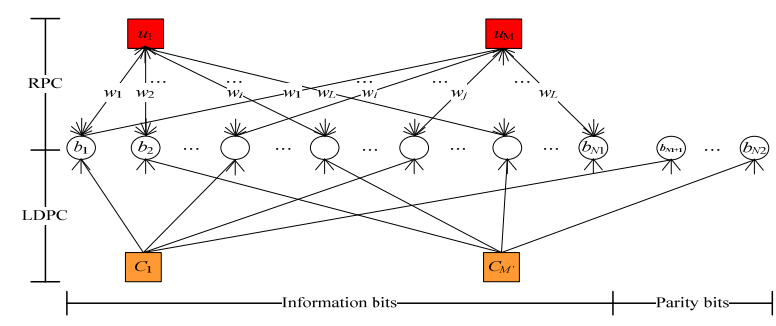

Figure 3. The diagram of joint decoding. 
In time slot $t_{3}$, after $\mathrm{D}$ received signal from relay node, $D$ performs LDPC decoding. $D$ receives the signal $\boldsymbol{Y}_{\boldsymbol{R} D}$ from $R$, and $\boldsymbol{Y}_{R D}$ can be presented by

$$
Y_{R D}=X_{R}+N_{S D}
$$

Next, $D$ assemble soft information from RPC decoder and soft parity information from relay node together, we get input data as following,

$$
Q_{j, i}^{1}(0)=\left[\hat{b}_{D}^{0}, Y_{0}\right] \text { and } Q_{j, i}^{0}(\mathbf{0})=\left[\hat{b}_{D}^{1}, Y_{1}\right]
$$

where

$$
Y_{1}(i)=\frac{1}{1+e^{-2 Y_{R D}(i) / \sigma_{R D}^{2}}}, \text { and } Y_{0}=1-Y_{1}
$$

\subsection{Analysis}

The scheme proposed in this paper has two important features. The first is that it realizes the rate adaptation at receiver. Due to using RPC decoding at relay node and destination node, our system will automatically decode the signal from source node according to the channel quality. As long as source node does not receive the ACK from destination node, it will keep broadcasting more and more modulation symbols to relay and destination node. With the increase of receiving symbols, the probability of RPC decoding success will improved. Otherwise, source node will send next data frame. With the help of LDPC encoding at relay node, the joint decoding performance in destination node is significantly improved. Thus, the number of demodulation symbols for RPC decoding can be dramatically reduced. In another words, joint decoding can decode successfully with much less symbols from source node with the help of relay. The second one is that joint decoding at destination node has two parts input, i.e., parity information from relay node and soft information from RPC encoder2. The joint decoding performance will be improved with the improvement of reliability of input data. Obviously, the reliability of soft information and parity information are improved with the help of relay node.

\section{Simulation Results}

In this section, we implement the decoding algorithm of RPC and LDPC algorithm, and use them to construct a relay system discussed in Section II. In our experiment, we select the SNR in SD link from $5 \mathrm{~dB}$ to $30 \mathrm{~dB}$, and think as usual the SNR in SR link and RD link are all better than SD link. Here we consider a special case in relay model where the distance proportion of relay node to source node, relay node to destination node and source node to destination node $\left(d_{S R}: d_{R D}: d_{S D}\right)$ is 7:3:1 and $3: 7: 1$. In addition we experiment the bits block size is $N=400$ and the numbers of parity bits generated in relay node is $100,50,25$ and 10 corresponding to the different LDPC coding rate $0.8,0.89,0.94$ and 0.99 respectively. Finally, we use goodput [18] and BER as evaluation metrics to evaluate the performance of our scheme. No matter what kinds of evaluation metric, we all assume that ACK is immediately available in our simulation.

Figure 4 compares the BER performance in different schemes. Here "S-D" is the original RPC algorithm, " Relay $_{R=0.8}$ ", “ $\operatorname{Relay}_{R=0.89}$ ”, “ Relay $_{R=0.94}$ " and " Relay $_{R=0.99}$ " is our scheme with different LDPC coding rate in relay node. From Figure 4, we can see that all these relay schemes are better than "S-D". And with the LDPC coding rate dropping the performance of our scheme is better and better. For example, as shown in Figure 4(a), when $d_{S R}: d_{R D}=3: 7$ and BER equal 10-5, our relay schemes have performance improved about $4 \mathrm{~dB}, 3.1 \mathrm{~dB}, 2.2 \mathrm{~dB}$ and $1.6 \mathrm{~dB}$ more than "S-D". In addition, we can find that there are the similar performances when $d_{S R}: d_{R D}=7: 3$ as shown in Figure 4(b). The main reason is that it is usually thought that the communication condition in SR link and RD link are all better than SD link, so the RPC decoding performance in relay node is better than destination node, which makes the correctness of RPC decoding in relay node all improved and the reliability of the parity bits similar in destination too. Thus, with the help of relay node, the whole system performance is less relation with the relay node's location when the LDPC rate in relay node is the same.

We also evaluate our scheme by good put metric in the actual wireless scenario. Here we set the bits block size is $N=400$ and the sending increasing step in source node is 10 modulation signals, the SNR range from $5 \mathrm{~dB}$ to $30 \mathrm{~dB}$ and $d_{S R}: d_{R D}=7: 3$. In order to test the performance of our scheme, we also consider the LDPC rate with 0.8 and 0.94 . Figure 5 shows the result where "S-D" is

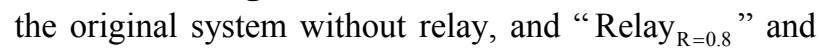
"Relay ${ }_{\mathrm{R}=0.94}$ " are our scheme. We can observe that our different schemes are all higher than "S-D" at least 0.38 bit $/ \mathrm{s} / \mathrm{Hz}$ and $0.28 \mathrm{bit} / \mathrm{s} / \mathrm{Hz}$ when SNR from $5 \mathrm{~dB}$ to $25 \mathrm{~dB}$. But all the throughputs are almost the same value 6.6 $\mathrm{bit} / \mathrm{s} / \mathrm{Hz}$ when SNR more than $25 \mathrm{~dB}$.Obviously, the reason we can achieve higher throughput is the relay's help. In addition, we also can observe that with the increasing of the LDPC rate, the goodput became higher and higher. The main reason is that the LDPC rate higher can provide more protection to information bits.

\section{Conclusions}

This paper investigates the scheme for parallel concatenation of LDPC and RPC. Though RPC perform the functionality of coding and modulation simultaneously, its 


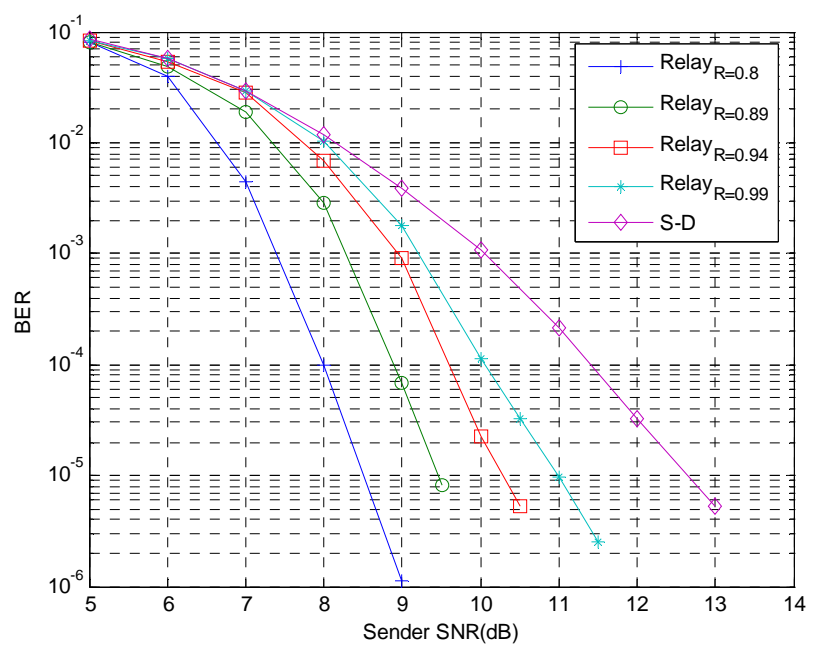

(a) $d_{S R}: d_{R D}=3: 7$

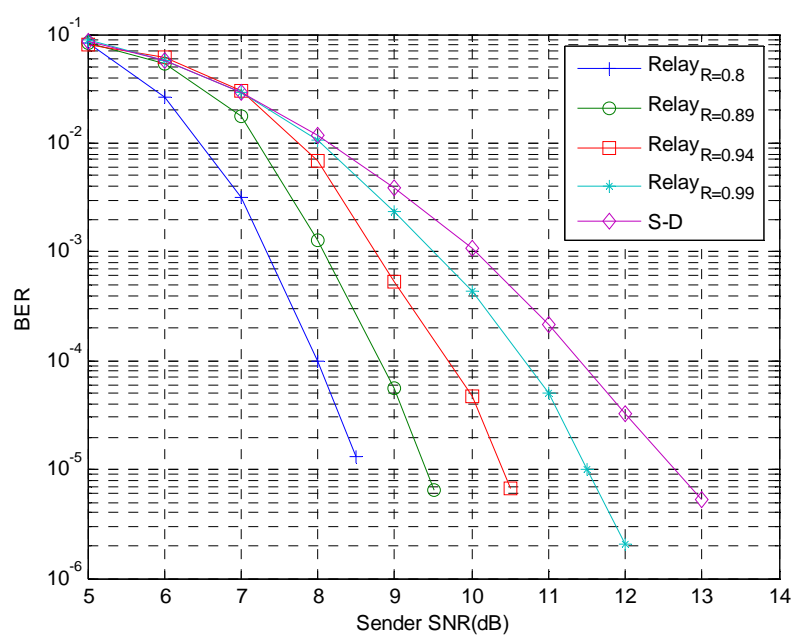

(b) $d_{S R}: d_{R D}=7: 3$

Figure 4. BER performance comparison among different schemes.

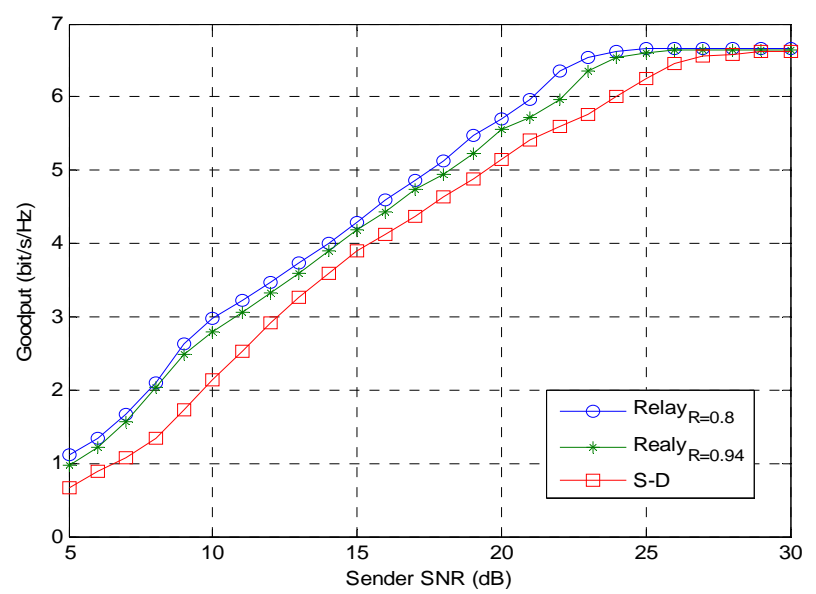

Figure 5. Throughputs comparison of SD link and different relay channel schemes.

coding performance is not good enough. As we expect, the concatenation of LDPC and RPC brings significant gain, e.g., coding gain $4 \mathrm{~dB}, 3.1 \mathrm{~dB}, 2.2 \mathrm{~dB}$ and $1.6 \mathrm{~dB}$ for coding rate $0.8,0.89,0.94$ and 0.99 respectively.

On the other hand, parallel concatenation structure fits the 3-node relay system well. In order to reduce overhead, we make use of distributed source code concept here, and relay node only sends parity code bits. The destination node uses the direct-link signal as side information, and relay-link signal to perform joint decoding. This scheme further improves rate adaptation feature of RPC. The efficient improvement of $0.3 \mathrm{bit} / \mathrm{s} / \mathrm{Hz}$ spectrum is obtained. Next step, we plan to investigate the joint code design of RPC and LDPC to optimize performance further.

\section{Acknowledgements}

The author wishes to thank the reviewers for their constructive comments which greatly improve the presentation of these results. This work is financially supported by NSFC General Program under contract No. 61173041.

\section{REFERENCES}

[1] Gudipati, Aditya, and Sachin Katti, "Automatic Rate Adaptation," Proceedings of the 9th ACM SIGCOMM Workshop on Hot Topics in Networks. ACM, 2010. Doi:10.1145/1868447.1868461

[2] J. D. Brown, S. Pasupathy and K. N. Plataniotis, "Adaptive Demodulation Using Rateless Erasure Codes," Communications, IEEE Transactions on, Vol. 54, No. 9, 2006. pp. 1574-1585.doi: 10.1109/TCOMM.2006.881236

[3] A. Forenza, R. W. Heath, Jr., "Link Adaptation and Channel Prediction in Wireless OFDM Systems," Circuits and Systems, 2002. MWSCAS-2002. The 2002 45th Midwest Symposium on, Vol. 3, 2002, pp. III211-214. doi: 10.1109/MWSCAS.2002.1187008

[4] H. Cui, et al., "Seamless Rate Adaptation for Wireless Networking," Proceedings of the 14th ACM International Conference on Modeling, Analysis and Simulation of Wireless and Mobile Systems. ACM, 2011. doi:10.1145/2068897.2068971

[5] Gudipati, Aditya, and S. Katti, "Strider: Automatic Rate Adaptation and Collision Handling," SIGCOMM-Computer Communication Review 41.4, 2011, p. 158.

[6] Perry, Jonathan, Hari Balakrishnan, and Devavrat Shah. "Rateless Spinal Codes," Proceedings of the 10th ACM Workshop on Hot Topics in Networks. ACM, 2011. doi: $10.1145 / 2070562.2070568$

[7] R. G. Gallager, "Low-density Parity-check Codes," Information Theory, IRE Transactions on, Vol. 8, No. 1, 1962, pp. 21-28, doi: 10.1109/TIT.1962.1057683

[8] D. J. C. MacKay and R. M. Neal, "Near Shannon Limit Performance of Low Density Parity Check Codes," Electronics Letters, Vol. 32, No. 18, 1996, pp. 
1645-1646. doi: 10.1049/el:19961141

[9] Azmi, M.H.; Jun Li; Jinhong Yuan; Malaney, R., "Soft decode-and-forward using LDPC coding in half-duplex relay channels," Information Theory Proceedings (ISIT), 2011 IEEE International Symposium on, vol., no., pp.1479-1483, July 31 2011-Aug. 52011 doi: 10.1109/ISIT.2011.6033787

[10] A. Chakrabarti, A. de Baynast, A. Sabharwal, B. Aazhang, "Low Density Parity Check Codes for the Relay Channel," Selected Areas in Communications, IEEE Journal on, Vol. 25, No. 2, 2007, pp. 280-291. doi: 10.1109/JSAC.2007.070205

[11] J. Hu, M. Du and T. M. "Low Density Parity Check Codes over Wireless Relay Channels," Wireless Communications, IEEE Transactions on, Vol. 6, No. 9, 2007, pp. 3384-3394.doi: 10.1109/TWC.2007.06083

[12] C. X. Li, G. S. Yue, X. D. Wang and M. A. Khojastepour, "LDPC Code Design for Half-Duplex Cooperative Relay," Wireless Communications, IEEE Transactions on, Vol. 7, No. 11, 2008, pp. 4558-4567. doi: 10.1109/T-WC.2008.070482

[13] M. H. Azmi, J. Li, J. H. Yuan and R. Malaney, "Soft Decode-and-forward Using LDPC Coding in Half-duplex Relay Channels," Information Theory Proceedings (ISIT), 2011 IEEE International Symposium on, 2011, pp. 1479-1483.doi: 10.1109/ISIT.2011.6033787

[14] P. Razaghi, W. Yu, "Bilayer Low-Density Parity-Check Codes for Decode-and-Forward in Relay Channels," Information Theory, IEEE Transactions on, Vol. 53, No. 10, 2007, pp. 3723-3739. doi: 10.1109/TIT.2007.904983

[15] P. Y. Tan, K. Xie and J. Li, "Slepian-Wolf Coding Using Parity Approach and Syndrome Approach," Information Sciences and Systems, 2007. CISS '07. 41st Annual Conference on , pp. 708, 713, 14-16 March 2007. doi: 10.1109/CISS.2007.4298397

[16] S. S. Pradhan, K. Ramchandran, "Distributed Source Coding Using Syndromes (DISCUS): Design and Construction," Information Theory, IEEE Transactions on, Vol. 49, No. 3, 2003, pp. 626-643. doi: 10.1109/TIT.2002.808103

[17] Garcia-Frias, Javier and Z. X. Xiong, "Distributed Source and Joint Source-channel Coding: From Theory to Practice," Proceedings of IEEE ICASSP. USA, Vol. 5, 2005, pp. 1093-1096.

[18] D. J. Qiao, S. Choi; K. G. Shin, "Goodput Analysis and Link Adaptation for IEEE 802.11a Wireless LANs," Mobile Computing, IEEE Transactions on, Vol. 1, No. 4, 2002, pp. 278,292. doi: 10.1109/TMC.2002.1175541 